\title{
Performance Analysis of Mobile Video Networks
}

\author{
Lingwei $\mathrm{Xu}{ }^{1,2}, \mathrm{Ye} \mathrm{Tao}^{1}, \mathrm{Ye} \mathrm{Li}^{2}$, Jingjing Wang ${ }^{1}$, Conghui $\mathrm{Cao}^{3}$, Changfeng Tian ${ }^{1}$
} \{gaomilaojia2009@163.com1,ye.tao@qust.edu.cn²,kathy1003@163.com ${ }^{3}$ \}

1.Department of Information Science and Technology, Qingdao University of Science and Technology, Qingdao 266061, China; 2.Qilu University of Technology (Shandong Academy of Sciences) ,Shandong Computer Science Center (National Supercomputer Center in Jinan), Shandong Provincial Key Laboratory of Computer Networks, Jinan, 250101, China; 3.College of Information Science and Engineering, Ocean University of China, Qingdao 266100, China;

\begin{abstract}
With the tremendous growth of mobile videos, mobile video communication is becoming increasingly important to influence users browsing and searching experience.In this paper, we present the approximate average symbol error probability (ASEP) and outage probability (OP) performance for mobile video networks. We derive the exact closed-form OP and ASEP expressions for amplify-andforward (AF) relaying. The analytical results match perfectly with the simulation results. Further, we evaluate the impact of power allocation on OP performance.
\end{abstract}

Keywords: mobile video networks; amplify-and-forward, average symbol error probability; outage probability;

\section{Introduction}

In recent years, mobile video communication has attracted significant attention from both academic and industrial fields [1]. Based semi-blind video watermarking algorithm, [2] proposed a robust block classification to enhance the robustness performance. In [3], the authors presented a framework to collect videos from smartphones in the public.

Cooperative diversity has been employed to improve the quality of service (QoS) for mobile communication.The authors applied the innovative multi-objective optimization methods to maximize the rate quality, and minimize the total transmission power [4]. The authors investigated performance analysis for an energy harvesting relay system with multiple co-channel interferences over Nakagami-m fading channels [5].

In $[4,5]$, the performance is only considered for Rayleigh and Nakagami-m fading channels. However, the effects of mobile video communication is far severe than what can be modeled using the Rayleigh and Nakagami-m fading channels [6].Therefore, the study on the performance of the mobile video networks is essential. In this paper, we derive the exact closed-form average symbol error probability (ASEP) and outage probability (OP) expressions for amplify-and-forward (AF) relaying. Furthermore, the theoretical results demonstrate excellent agreement with Monte-Carlo simulation results, which validates our analysis.

The rest of the paper is organized as follows. The mobile video networks model is presented in Section 2. We have investigated OP and ASEP performance in Section 3,4. Section 5 conducts Monte-Carlo simulations to verify the analytical results. Finally, we conclude the paper in Section 6 .

\section{The System Model}

Figure 1. shows mobile video transmission networks model. With the help of $L$ single- 
antenna mobile relay (MR) nodes, the mobile source (MS) communicates with mobile destination (MD) node.

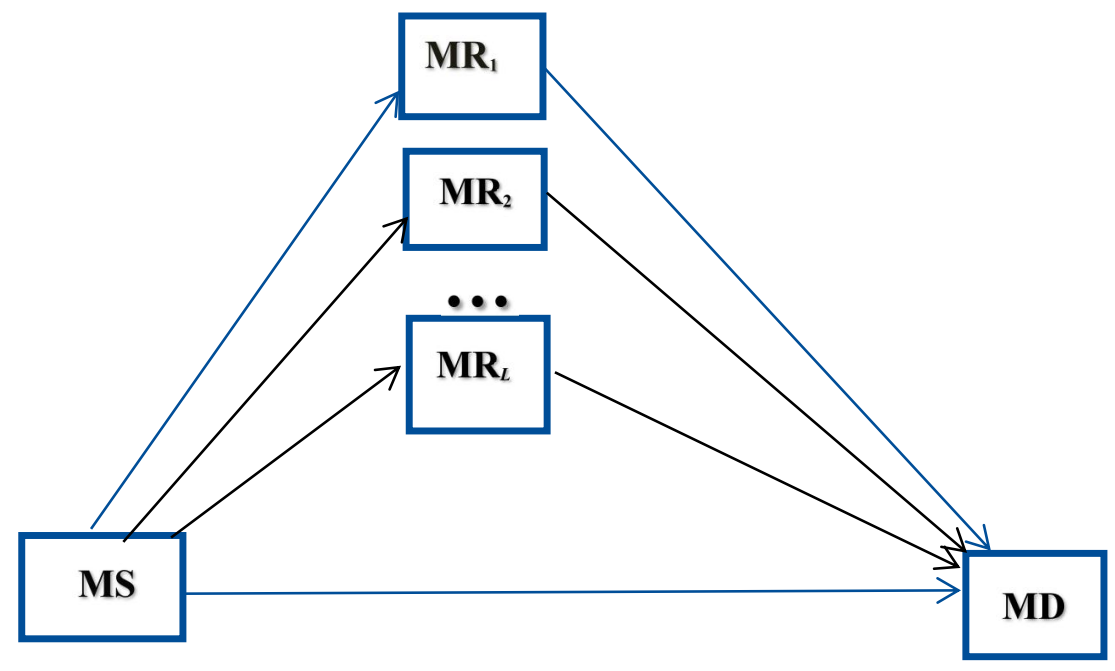

Fig. 1. The system model

We use $h=h_{z}, z \in\{\mathrm{SD}, \mathrm{SR} l, \mathrm{RD} l\}$, to represent the complex channel coefficients. $h$ follows $N$-Nakagami distribution[6].The total transmission energy is $E$.

MS transmits the video signal $x$ in the first time slot. $\mathrm{MD}$ and $\mathrm{MR}_{l}$ receive video signals as

$$
\begin{gathered}
r_{\mathrm{SD}}=\sqrt{K E} h_{\mathrm{SD}} x+n_{\mathrm{SD}} \\
r_{\mathrm{SR} l}=\sqrt{G_{\mathrm{SR} l} K E} h_{\mathrm{SR} l} x+n_{\mathrm{SR} l}
\end{gathered}
$$

where the relative gain of the $\mathrm{MS} \rightarrow \mathrm{MD}$ link is $G_{\mathrm{SD}}=1, G_{\mathrm{SR} l}$ is the relative gain of $\mathrm{MS} \rightarrow \mathrm{MR}_{l}$, the mean and variance of $n_{\mathrm{SR} l}$ and $n_{\mathrm{SD}}$ are 0 and $N_{0} / 2 . K$ is the power-allocation parameter.

In the second time slot,we only select the best MR to participate in cooperation

$$
\gamma_{\mathrm{R}^{*}}=\max _{1 \leq l \leq L}\left(\gamma_{l}\right)
$$

where

$$
\begin{gathered}
\gamma_{l}=\frac{\gamma_{\mathrm{SR} l} \gamma_{\mathrm{RD} l}}{1+\gamma_{\mathrm{SR} l}+\gamma_{\mathrm{RD} l}} \\
\gamma_{\mathrm{SR} l}=\frac{K G_{\mathrm{SR} l}\left|h_{\mathrm{SR} l}\right|^{2} E}{N_{0}}=K G_{\mathrm{SR} l}\left|h_{\mathrm{SR} l}\right|^{2} \bar{\gamma} \\
\gamma_{\mathrm{RD} l}=\frac{(1-K) G_{\mathrm{RD} l}\left|h_{\mathrm{RD} l}\right|^{2} E}{N_{0}}=(1-K) G_{\mathrm{RD} l}\left|h_{\mathrm{RD} l}\right|^{2} \bar{\gamma}
\end{gathered}
$$

MD receives the signal as

$$
r_{\mathrm{R}{ }^{*} \mathrm{D}}=\sqrt{c E} h_{\mathrm{SR}^{*}} h_{\mathrm{R}{ }^{*} \mathrm{D}} x+n_{\mathrm{DD}}
$$

where the mean and variance of $n_{\mathrm{DD}}$ are 0 and $N_{0} / 2$. $c$ is given as [7] 


$$
c=\frac{K(1-K) G_{\mathrm{SR}^{*}} G_{\mathrm{R}^{*} \mathrm{D}} E / N_{0}}{1+K G_{\mathrm{SR}^{*}}\left|h_{\mathrm{SR}^{*}}\right|^{2} E / N_{0}+(1-K) G_{\mathrm{R}^{*} \mathrm{D}}\left|h_{\mathrm{R}^{*} \mathrm{D}}\right|^{2} E / N_{0}}
$$

MD uses the selection combining (SC) scheme and receives the SNR as

$$
\gamma_{\mathrm{SC}}=\max \left(\gamma_{\mathrm{SD}}, \gamma_{\mathrm{SRD}}\right)
$$

where

$$
\begin{gathered}
\gamma_{\mathrm{SD}}=\frac{K\left|h_{\mathrm{SD}}\right|^{2} E}{N_{0}}=K\left|h_{\mathrm{SD}}\right|^{2} \bar{\gamma} \\
\gamma_{\mathrm{SRD}}=\frac{\gamma_{\mathrm{SR}^{*} \gamma_{\mathrm{R}}{ }^{*} \mathrm{D}}}{1+\gamma_{\mathrm{SR}^{*}}+\gamma_{\mathrm{R}}{ }^{*} \mathrm{D}}=\max _{1 \leq l \leq L}\left(\gamma_{l}\right)
\end{gathered}
$$

With the aid of [8], we can obtain an upper bound $\gamma_{\text {up }}$ as

$$
\gamma_{\mathrm{SRD}}<\gamma_{\mathrm{up}}=\min \left(\gamma_{\mathrm{SR}^{*}}, \gamma_{\mathrm{R}^{*} \mathrm{D}}\right)=\max _{1 \leq l \leq L}\left(\min \left(\gamma_{\mathrm{SR} l}, \gamma_{\mathrm{RD} l}\right)\right)
$$

\section{The OP of Mobile Video Networks}

We obtain the cumulative density functions (CDF) of $\gamma_{\text {up }}$ as

$$
F_{\gamma_{\mathrm{up}}}(r)=\prod_{l=1}^{L}\left(\begin{array}{l}
\frac{1}{\prod_{t=1}^{N} \Gamma\left(m_{t}\right)} G_{1, N+1}^{N, 1}\left[\left.\frac{r}{\overline{\gamma_{\mathrm{SR} l}}} \prod_{t=1}^{N} \frac{m_{t}}{\Omega_{t}}\right|_{m_{1}, \ldots, m_{N}, 0} ^{1}\right] \\
+\frac{1}{\prod_{t t=1}^{N} \Gamma\left(m_{t t}\right)} G_{1, N+1}^{N, 1}\left[\left.\frac{r}{\overline{\gamma_{\mathrm{RD} l} l}} \prod_{t t=1}^{N} \frac{m_{t t}}{\Omega_{t t}}\right|_{{ }_{1}, \ldots, m_{N}, 0} ^{1}\right] \\
-\frac{1}{\prod_{t=1}^{N} \Gamma\left(m_{t}\right) \prod_{t t=1}^{N} \Gamma\left(m_{t t}\right)} G_{1, N+1}^{N, 1}\left[\left.\frac{r}{\overline{\gamma_{\mathrm{SR} l}}} \prod_{t=1}^{N} \frac{m_{t}}{\Omega_{t}}\right|_{m_{1}, \ldots, m_{N}, 0} ^{1}\right] \\
\times G_{1, N+1}^{N, 1}\left[\left.\frac{r}{\overline{\gamma_{\mathrm{RD}} l}} \prod_{t t=1}^{N} \frac{m_{t t}}{\Omega_{t t}}\right|_{m_{1}, \ldots, m_{N}, 0} ^{1}\right]
\end{array}\right)
$$

where $G[\cdot]$ is the Meijer's G-function [6].

The CDF of $\gamma_{S D}$ is given as

$$
F_{\gamma_{\mathrm{SD}}}(r)=\frac{1}{\prod_{i=1}^{N} \Gamma\left(m_{i}\right)} G_{1, N+1}^{N, 1}\left[\left.\frac{r}{\overline{\gamma_{\mathrm{SD}}}} \prod_{i=1}^{N} \frac{m_{i}}{\Omega_{i}}\right|_{m_{1}, \ldots, m_{N}, 0} ^{1}\right]
$$

We obtain the approximate $\mathrm{OP}$ as

$$
F_{\gamma_{\mathrm{SCA}}}\left(r_{\mathrm{th}}\right)=F_{\gamma_{\mathrm{SD}}}\left(r_{\mathrm{th}}\right) F_{\gamma_{\mathrm{tu}}}\left(r_{\mathrm{th}}\right)
$$

where $r_{\text {th }}$ is a given threshold. 


\section{The Approximate ASEP of Mobile Video Networks}

With the aid of [9], the approximate ASEP is given as

$$
P=\frac{a \sqrt{b}}{2 \sqrt{\pi}} \int_{0}^{\infty} \frac{\exp (-b r)}{\sqrt{r}} F_{\gamma_{\mathrm{SCA}}}(r) \mathrm{d} r
$$

where the different modulation types determine the parameters $a$ and $b$.

We obtain the approximate ASEP as

$$
P=\frac{a \sqrt{b}}{2 \sqrt{\pi}} G
$$

where

$$
\begin{aligned}
& G=\int_{0}^{\infty} \frac{1}{\sqrt{r}} G_{0,1}^{1,0}\left[\left.b r\right|_{0} ^{-}\right] G_{1, N+1}^{N, 1}\left[\left.\frac{r}{\overline{\gamma_{\mathrm{SD}}}} \prod_{i=1}^{N} \frac{m_{i}}{\Omega_{i}}\right|_{m_{1}, \ldots, m_{N}, 0} ^{1}\right]
\end{aligned}
$$

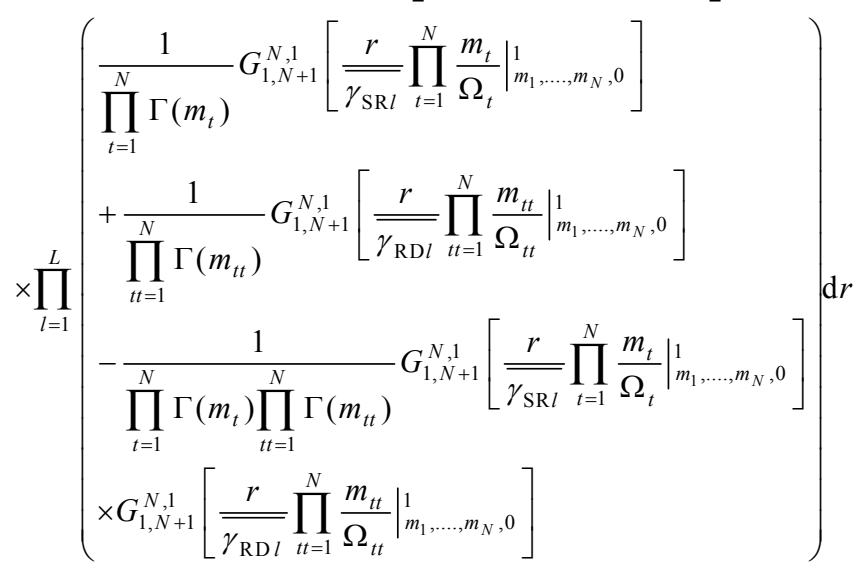

\section{Numerical Results}

In this section, we define $\mu=G_{\mathrm{SU}} / G_{\mathrm{RU}}$ as the relative geometrical gain. $E=1$.

Figure 2 presents the ASEP performance versus $L$. Here, QPSK is used. $L=1,2,3$, $\mu=0 \mathrm{~dB}, K=0.5, N=2, m=1$. We can find that the Monte-Carlo simulation results match perfectly with the theoretical results. Increasing $L$ improves the ASEP performance. For example, when SNR $=12 \mathrm{~dB}$, the ASEP is $6.6 \times 10^{-2}$ with $L=1,2.8 \times 10^{-2}$ with $L=2,1.3 \times 10^{-2}$ with $L=3$. 


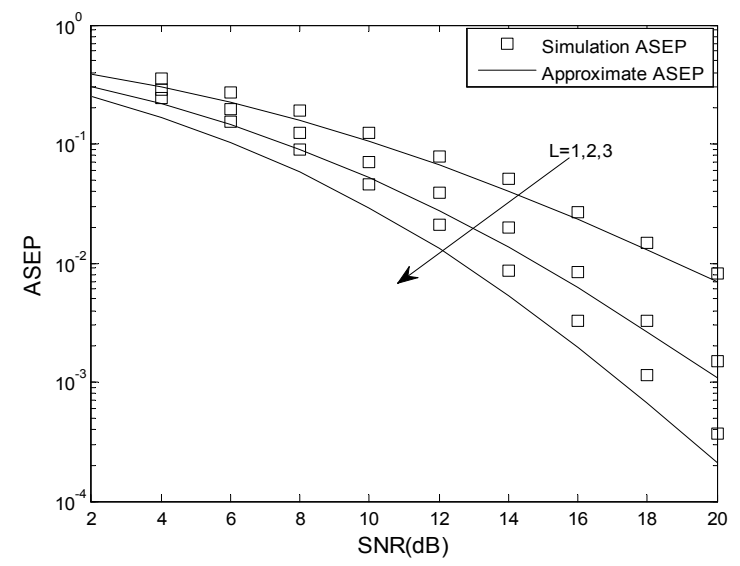

Fig. 2. The ASEP performance versus $L$

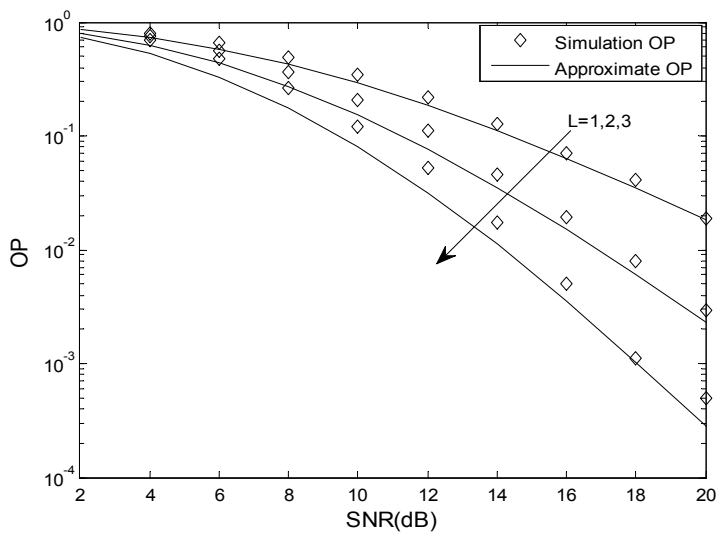

Fig. 3. The OP performance versus $L$

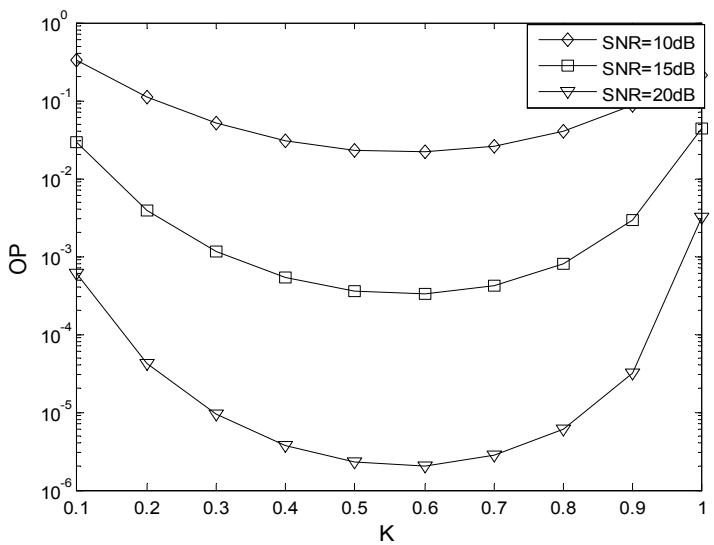

Fig. 4. The OP performance versus $K$

Figure 3 presents the OP performance versus $L$. $L=1,2,3, \mu=0 \mathrm{~dB}, K=0.5, N=2, m=1$, $r_{\mathrm{th}}=4 \mathrm{~dB}$. From Fig. 3 , it is observed that the Monte-Carlo simulation results match perfectly 
with the theoretical results. Increasing $L$ improves the OP performance. For example, when $\mathrm{SNR}=14 \mathrm{~dB}$, the $\mathrm{OP}$ is $1.1 \times 10^{-1}$ with $L=1,3.5 \times 10^{-2}$ with $L=2,1.1 \times 10^{-2}$ with $L=3$.

In Figure 4, the OP in (15) is plotted as a function of $K . N=2, m=2, \mu=0 \mathrm{~dB}, L=2, r_{\mathrm{th}}=4 \mathrm{~dB}$. Here, we use Golden-Section search method to find the optimum power allocation (OPA) values of $K$. For example, $K=0.58$ with $\mathrm{SNR}=10 \mathrm{~dB} ; K=0.58$ with $\mathrm{SNR}=15 \mathrm{~dB} ; K=0.58$ with $\mathrm{SNR}=20 \mathrm{~dB}$.

\section{Conclusions}

In this paper, we had investigated the performance of the AF relaying mobile video networks. The simulation results show that $L$ and $K$ have a significant influence on the ASEP and OP performance of mobile video networks.

Acknowledgments.This project was supported by Open Research Fund from Shandong provincial Key Laboratory of Computer Networks(no.SDKLCN-2017-01), Shandong Province Natural Science Foundation(no.ZR2017BF023),China Postdoctoral Science Foundation funded project(no.2017M612223), Shandong Province Postdoctoral Innovation Project(no. 201703032).

\section{References}

[1] Chen,S., and Zhao,J., "The requirements, challenges and technologies for 5G of terrestrial mobile telecommunication,"IEEE Communications Magazine, Vol. 52, No. 5, pp. 36-43(2014).

[2] Bahrami,Z., Tab,F. A.”A new robust video watermarking algorithm based on SURF features and block classification," Multimedia Tools and Applications,Vol. 77, No. 1, pp. 327-345(2018).

[3] Bohez,S.,Daneels,G.,Herzeele,L.V.,et al."The crowd as a cameraman: on-stage display of crowdsourced mobile video at large-scale events," Multimedia Tools and Applications, Vol. 77, No. 1,pp. 597-629(2018).

[4] Soleimanpour-moghadam, M., Talebi,S."Relay selection and power allocation for energy-efficient cooperative cognitive radio networks." Physical Communication, Vol.28,No.6,pp. 1-10(2018).

[5] Alodeh,M., Chatzinotas,S., Ottersten,B. "Performance analysis of energy harvesting AF relay systems over Nakagami-m fading channels with co-channel interferences,"Physical Communication, Vol. 27, No.4, pp.86-92(2018).

[6] Karagiannidis,G. K., Sagias,N. C., and Mathiopoulos,P. T., "N*Nakagami: a novel stochastic model for cascaded fading channels," IEEE Transactions on Communications, Vol. 55, No.8, pp.1453-1458(2007) .

[7] Li, Z.X., Jia,L.Z.,Li, F.and Hu,H.Y., "Outage performance analysis in relay-assisted inter-vehicular communications over double-Rayleigh fading channels," Proceedings of the IEEE International Conference on Communications \& Mobile Computing, Shenzhen, China,pp. 266-270(2010).

[8] Ochiai, H., Mitran, P. and Tarokh, V., "Variable-Rate Two-Phase Collaborative Communication Protocols for Wireless Networks," IEEE Transactions on Vehicular Technology, Vol. 52, No. 9, pp. 4299-4313(2006).

[9] Anghel,P. A., and Kaveh,M. "Exact symbol error probability of a cooperative network in Rayleigh-fading environment," IEEE Transactions on Wireless Communications, Vol. 3, no. 5, pp. 1416-1421(2004). 